\title{
Knowledge Transmission among Preservation Practitioners in Dubai
}

\author{
Adil M. Abdalla
}

\begin{abstract}
The economic importance and strategic position of the Middle East have epistemologically twisted most of its true cultural recognition. Both late urban definition and mutual communications have crippled some of its Bedouin societies from proving their participation in global culture. Each society has some to share in human accumulative know-how, but geo-political environments and barriers have lots to do in such mutuality. Many investigations have proved effective transmission of knowledge, but also pointed out black and mysterious zones within the societies themselves. The main obstacle is generated from the oral Arabic culture itself, when episteme is conditioned with biological memory, synchronized interchange and ethical referrals.
\end{abstract}

The necessity of scientific and standard approaches of archeological research and architectural preservation exist and is proven to be of value in preservation of architectural knowledge. However, it is distinctly a hard task to use such standards to challenge the fast urbanization and social developments of our time.

While archeological research is apart from contemporary explanations, preservation research is proposed as being more important. Investigations on building concepts require in-depth analysis of habits and behaviors of both craftsmen and beneficiaries, which is a folkloric approach. The lack of linguistic research blocks sufficient use of traditional poetry, literature and folklore to reproduce evidence of technologies from the past. Obviously, the limited natural resources in the Arabian Peninsula have simplified both the architectural setting and construction methodologies and hidden the natural creativity or know-how of earlier societies. Unique architectural contributions need not be limited to definitions of monumental constructs or other impressive achievements. Rather, architecture is defined mainly by cultural and epistemological expressions. This is important in discovering the continuity and distribution of societies in human and global civilization. It is also important to include Middle Eastern architecture in the ongoing intensive identification of architectural perspectives.

Despite the late attention that has been given to preservation activities in Arabic States in the Persian Gulf, it is time to start assessment and evaluation of architectural traditions to challenge the remarkably fast development that is occurring. Various organizational, social and political factors are involved in the few serious attempts that have been made. Interventions by ambitious ruling powers have alternatively supported or thwarted such efforts. Lessons can be learned from these examples that highlight effective approaches useful in maintaining the immovable heritage elements in underdeveloped countries. These elements contain most of the remaining important and creative features of human architectural inputs that now benefit most of the globe. International involvement may be drawn to fill scientific gaps and requirements, while paths for serious cooperation are blocked with either hesitation or insufficient awareness of the matter.

\section{Correspondence}

Adil M. Abdalla, ICOMOS, ICOM, P O Box 29161, Dubai, UNITED ARAB EMIRATES.

adilsud@hotmail.com

Ethnobotany Research \& Applications 3:123-126 (2005) 
Bridging the true knowledge needs not only willingness, but also serious identification of both obvious and unconscious motivations that rule decision-making and practice. The conclusions may support the ongoing development of methodologies and professional practices.

\section{Introduction}

Indigenous knowledge is usually connected to sustainable development that focuses on particular areas of activities where it plays crucial roles in the socio-economic structure of the society that uses it. The role of indigenous knowledge are ignored in other sectors where modern technological parameters have shaped the processes and social structures instead. Generally, indigenous knowledge may be considered as the foundation of humanitarian research and applications that target preservation of core characteristics of a human civilization. One of the areas where hidden indigenous knowledge is involved is the preservation of architectural heritages. Preservation in this case involves a combination of both architecture and construction epistemologies.

Architecture is an application of artistic and functional attitudes, while construction is a usage of environmental and domestic resources. The main purpose of preservation is to maintain the evidence of human skills and production that made the built environment. These evidences contain concepts, knowledge and technologies that were prominent during certain periods of time in particular locations. Preserving these evidences requires keeping them free of any modern interventions. Such actions require conclusive awareness and understanding of traditional construction techniques with appropriate analysis of materials, structural behavior and workmanship. Preservation training programs have to include resources of indigenous knowledge, which enable convenient applications. Unfortunately changing social and cultural structures have affected the continuity of knowledge transmission in the absence of integrative educational systems .

\section{Knowledge}

The architectural profession has its own unique perspectives about the combination between physical and spiritual practices. It may be seen as opening the narrow perspective of the masses in ways that produce integration between masses and their motivations and affections. It links the scattered components of consciousness and gathers them to form epistemic realizations. This is directly linked with the storage of cultural principles of creativity. Therefore, architectural education has made verity of approaches as supporting tools to build the required knowledge for architects to re-shape our sculptured and visual world. Syntheses on art, history and management are crucial to form the background for an architect in any community. Like most professions, architecture is an ac- cumulative one, including various cultural aspects and findings that have led to development of its theories and applications.

One of the major distinctions between Greek and Persian schools is the weight of documentation, which enabled Greek (Western) civilization to become academic, while the Persian (Eastern) civilization remained mainly folkloric. This does not decrease the value of Eastern cultural contributions to human heritage, but provides and opportunity to integrate and to establish the harmony and balance that exists between them. Seats of influence were exchanged between Western and Eastern communities because of many religious, mythical and pragmatic movements. Arabs were caught in the middle and is reflected in their cultural productions and heritage characteristics. This may explain why Arabs are structurally imbalanced compared with neighboring nations in Persia, India, Turkey and Sub-Saharan Africa. While social stability is likely confirmed across their boarders, extreme reactions and social modifications have addressed the Arabic productions for the last fifty years. Historical weight, economic wealth and geopolitical factors did not shield the Arabic societies, but uprooted some aspects of their contemporary culture in favor of external influences.

\section{Arabic Preservation}

The first official Arabic activity in architectural preservation began in 1896 when significant numbers of Egyptian Islamic monuments began to be maintained. Other Arab countries subsequently started their organized activities after World War Two, while others still have yet to begin to take appropriate actions. New trends in maintaining architectural heritages accompany recently developed attitudes that include cultural tourism in economic plans. Accordingly, new locations were introduced to global tourism maps, e.g., Oman, Yemen and Dubai, while Saudi Arabia and Bahrain targeted inter-Arabian tourism and entertainment. Generous funds were granted for preservation in most Arab countries, supported by international professional and academic institutions that are interested in Arabic heritage and culture for various reasons.

Some Arab countries have unfortunate economic balances. Fortunately, these states have substantial national manpower that they are able to use to maintain their national monuments and preserve the traditional techniques of construction. The poverty that has monopolized low-income social layers has also preserved traditional crafts, such as masonry, carpentry, iron mongery, etc. The end result has been that authenticity has been maintained within the principles of architectural preservation employed.

Dubai, like many oil producers in Arabian Peninsula (GCC countries), has had to use foreign manpower to satisfy construction needs that are not met by its own profession- 


\section{Abdalla - Knowledge Transmission among Preservation Practitioners in Dubai 125}

als and young generations. UAE nationals are only $19 \%$ of the nations' residents and only $7 \%$ of its workforce. Among the 525,000 UAE students in formal education, only $0.3 \%$ are enrolled in vocational schools. At the same time Higher Colleges of Technology have no construction related programs for any of their 15,000 students. The result is that non-local construction inputs have impacted both common and preservation construction activities.

\section{Builder or Architect}

The acknowledgement made by the famous Aga Khan Award of Architecture in 1983 to Mr. Ahmed Hassan of Egypt has significantly declared the role of Arabic Builders in shaping Arabic Architecture. Examples of the work of this poorly literate and old mason are repeatedly recognized all over Arabic societies. Unfortunately, the dilemma between Traditionalism and Modernism was widely spread and marked the post-World War Two era. Modernism was and still does use much skill and capability to circumvent the principles of traditional methodologies. Indigenous knowledge needs to provide its own empirical and mathematical modules to preserve its seat in new hierarchies. In the current world, imitating Western societies, which have trained the new breed of teachers, professionals and decision-makers, have destroyed the cultural and ethical basis that Arab societies had established long ago. Pro-Western influence is widely appreciated as symptomatic of serious developments, which have labeled Traditionalism as synonymous with weakness and colonization. Traditional educational and training courses have been abandoned, while formal and vocational ones were greeted as the modern way.

Some Arabic scholars have shyly expressed concern about the growing conflicts that new architects confront in considering volumes and scales. No attempts have been recorded to involve the traditional building Masters in any sort of knowledge transmission to modern architects. The knowledge itself has thus turned from practical and accumulative processes to insufficient written materials and elegant or artistic drawings. This humiliation of the traditional Masters has been accompanied with poor documentation of practices thus blocking the few sincere attempts of recovery and use traditional knowledge. Theoretical debates have been used as media fuel rather than public guidance. Ivory tower scholars have promoted themselves rather than their ideas, which have been empowered with new political orders and agendas. The flood of new (foreign) architectural concepts has washed away the traditional construction memory. Now, a growing concern has marked the last twenty years that is focused on theoretical not practical or site grounds.

\section{Dubai}

Although the settlement of Dubay town is known from 1799, no maps have identified the settlement before
1819. Dubai is a tiny geographical, political and demographic composition that escaped the narrow frames of oil reserves and established its own name among the growing Arabic economic and commercial powers. Dubai has its own flavor of cultural development. Its was founded on stable ground, which reflects its distinct and tight political formula. This has prevented the common troubles of social development and seeded the ground for rapid growth. Since its development has been based on multiethnic manpower, many issues are ignored and others are debatable in a different manner than in other Arabic societies. Amazing initiatives were chosen to address its presence and ambition during the last five years. Both Western and Eastern epistemological schools are observed in Dubai applications, rational assessments and ambitious planning. While Dubai generously laid the foundations to join the growing southern IT chain, it wildly competes with older markets around the historically important economic center of the Middle East. Cultural initiatives have not been forgotten; hence they decorate the cake and attract further buyers. Dubai was and stills is a commercial center that inspires and teases its neighbors, despite their capabilities and potentials.

The trend of preservation was inaugurated in the Gulf area in mid 1980s, when early national professionals came back home from their Western schools and found themselves surrounded with history-appreciated environments. Sheikh Saied House was the first to be preserved in Dubai, using a "Gulf Methodology", where it was entirely demolished and rebuilt with modern materials and finally decorated with traditional materials. This work has earned the award of the Arab Cities Organization, which similarly represents both Arabic political and cultural trends. The massive demolition of $70 \%$ of the traditional districts of Dubai in 1990 let to the formation of the Dubai Municipality's Unit for the Restoration of Archeological Buildings in 1992 . The 15 man unit developed into a 300 man Historical Buildings Section by 1996 with annual budget of US $\$ 7,000,000$. However, only 3 employees had previous preservation track records. By 2000, Dubai had earned another 4 regional awards on its preservation activities, doing so with no international capacities applied.

\section{Knowledge Transmission}

As surveyed in 2000 , only $29 \%$ of Dubai preservation office-staff are UAE nationals; a senior manager, an architect, an engineer, 2 interior designers, 2 historians and 3 clerks. (Another 2 architects resigned in late 1998 because they were not satisfied with their employment.) No UAE national is involved in practical aspects of construction. None of these individuals had experienced the known site hazards that are familiar to construction practitioners. None of them had chosen preservation as a profession nor joined the Historical Buildings Section of Dubai Municipality. None of these individuals were older than their late twenties except the manager and the historians. Also, 
joining this activity was the first employment for $80 \%$ of them. Employment satisfaction was remarkably poor, among most of HBS staff, despite choosing their senior manager to receive Dubai Excellence Award for Governmental Performance in 1998.

Most of the 334 craftsmen, labors and helpers in HBS are Indians or Pakistanis. This matches the general demographic statistics of UAE, where $50 \%$ of residents are from the Indian Subcontinent. Recruitment does not target previous restoration experience; hence both availability and assessment tools are not applicable. A 1998 mission was assigned to an international consultant to assess and upgrade the organization. This failed and was dramatically terminated. Most of the projects that were surveyed were designed and constructed in-house hence in 1998 the awards to independent consultants and contractors were fought. This has resulted in unauthentic construction techniques that prevent standardization or international recognition despite the estimated US\$75 million spent during 1985-2000. Generally, none of Arabian architectural monuments are listed in World Heritage Lists except Bahla Forte in Oman.

\section{Transmission Pathways}

Limited success can describe the few pathways that HBS maintained in the area of knowledge transmission. $37 \%$ of these paths were conducted with professionals, while only $15 \%$ targeted elders. The majority of HBS staff had no gains of knowledge through attempts to learn traditional knowledge. Despite the availability of a US $\$ 200,000$ hitech facility for audio-visual documentation little was done to use this for collection and transmission of traditional knowledge. The single documentation specialist had resigned with no other remaining to take on the task.

\section{Learning from Elders}

Only a few meetings have been organized with some of the Dubai elders who have been involved in the construction business. Their talks that have been recorded are focused on materials, settings and construction procedures. The knowledge documentation methods used were extremely experimental; hence no guidelines or codes were generated to help develop better applications or scientific implementations. No programs or plans have been set in place to address these concern, although an ex-UAE University lecturer was appointed to run the research work of learning from elders.

\section{Design Terms of References}

The approved Terms Of References for restoration design have included a basic section for surveys, which includes historical, visual and architectural documentation. The historical survey aims to collect all historical and docu- mental data of the building, e.g., owners, masons, coincidences of construction and the accompanied socio-cultural environment. The implementations by either independent consultants or HBS staff do not focus on indigenous knowledge or traditional workmanship. Most of the reports focus instead upon biographies of owners or tenants.

\section{Random Explorations}

A series of visits has been made to local locations and organizations to explore the procedures employed in restoration projects. In each case, the head of section solely and personally had arranged most of these visits, which have not been converted into any sort of documentation, beyond souvenir photographs.

\section{Conclusions}

This paper was prepared to highlight the importance of indigenous knowledge in preservation practices. There are certainly many other examples that are believed to be elsewhere in the world that would illustrate the need for in-depth analysis and exposure of the problems of restoration of ancient architecture using traditional knowledge. The assumed results of analyses of traditional architectural methods will not only focus on better practices for preservation, but also will encourage the application of indigenous knowledge in other areas of research.

The architectural heritage of the world should be of interest to all mankind. Hence proper documentation of our roots is essential if we are to have a clear forecast for our future. False applications of architectural knowledge hurt the purity and sincerity that we try to teach our children. Any political influence on scientific applications has to have limits and ceilings to keep things in acceptable order. The coming generations will have tougher verdicts on our actions, so it is appropriate to seek their appreciation now rather than forgiveness later.

Therefore, the international scientific and professional institutions concerned with heritage and preservation have to be addressed to seek appropriate means to include indigenous knowledge in their architectural concepts, guidelines, manuals and codes of practice, to clearly confirm its crucial role in the true history of mankind.

Finally, many communities are advised to re-organize their vocational training systems to assure that the role of indigenous knowledge is not lost. This should be intended to preserve cultural distinctions as core parts of local sustainability and welfare. 